\title{
New Results on Bottom Baryons with the CDF II Detector
}

\section{Constantino Calancha}

(on behalf of the CDF Collaboration)

CIEMAT, Madrid, Spain

E-mail: Calanchaefnal.gov

\section{Igor V. Gorelov*}

(on behalf of the CDF Collaboration)

University of New Mexico, Albuquerque, USA

E-mail: gorelovefnal.gov

\begin{abstract}
We present measurements of the masses and widths of four bottom baryon resonances, $\Sigma_{b}^{(*) \pm}$, reconstructed in the $\Lambda_{b}^{0} \pi^{ \pm}$hadron decay modes. The isospin mass splittings for the $\Sigma_{b}$ and $\Sigma_{b}^{*}$ multiplets are extracted as well. The analysis is based on a data sample corresponding to an integrated luminosity of $\int \mathscr{L} d t \approx 6.0 \mathrm{fb}^{-1}$.
\end{abstract}

35th International Conference of High Energy Physics - ICHEP 2010,

July 22 - 28, 2010,

Paris, France

\footnotetext{
* Speaker.
} 
The heavy baryons with a single heavy quark are the helium atoms of QCD with nucleus as a heavy quark $Q$ and two orbiting electrons as a light di-quark $q_{1} q_{2}$. The heavy quark in the baryon may be used as a probe of a confinement which at least will allow us to study a non-perturbative QCD somewhat deeper than we do it with light baryons. In the experimental analysis presented below we investigate bottom baryon states $\Sigma_{b}, \Sigma_{b}^{*}$ with the quark content $b\left\{q_{1} q_{2}\right\}$, whereof a heavy quark spin $S_{b}=\frac{1}{2}^{+}$and a spin of the flavor symmetric di-quark $S_{\left\{q_{1} q_{2}\right\}}=1^{+}$constitute two isospin $I=1$ triplets with the total spin $J^{P}=\frac{1}{2}^{+}$and $J^{P}=\frac{3}{2}^{+}$, see Fig. 1.

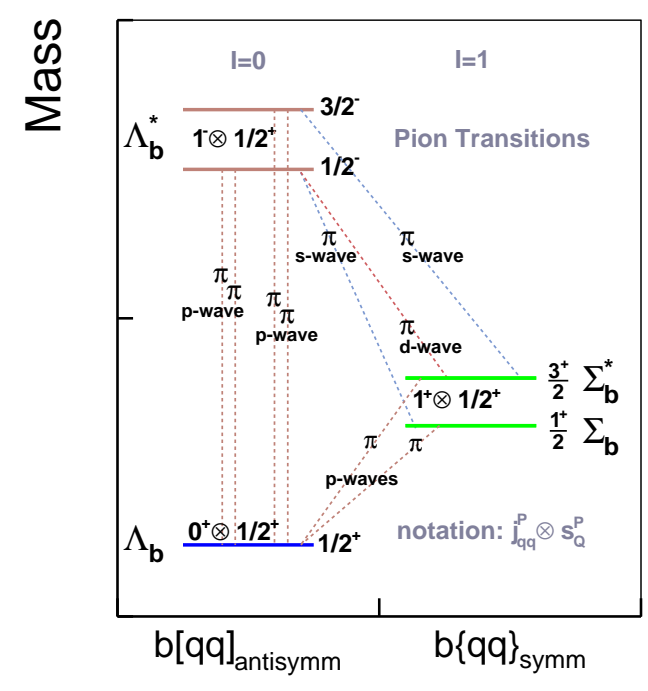

Figure 1: The pion transitions of $\Sigma_{b}^{(*)}$ states and a first orbital P-wave excitations $\Lambda_{b}^{* 0} . \Sigma_{b}^{(*)}$ states are the lowest-lying $S$ - wave states which can decay to the $J^{P}=\frac{1}{2}^{+}$singlet $\Lambda_{b}^{0}$ via strong processes involving soft pion emissions provided sufficient phase space is available for a given mode.

This study follows a discovery of the $\Sigma_{b}^{(*)}$ states made by CDF Collaboration in 2006 [1]. We intend to confirm the first observation of those states using a larger data-sample. Fitting the mass difference spectra of $\Sigma_{b}^{(*)+}$ and $\Sigma_{b}^{(*)-}$ independently and with floating background model parameters, we aim to measure masses, intrinsic natural widths and isospin mass splittings within $\Sigma_{b}$ and $\Sigma_{b}^{*}$ iso-triplets. With our measurements we will provide the next input to the theoretical community initiating the new round of heavy baryon calculations including somewhat newer tasks like e.g. natural width estimates.

Some recent HQET calculations for bottom baryons are done in [四]. In potential quark model the mass differences like $\Sigma_{Q}-\Lambda_{Q}, \Sigma_{Q}^{*}-\Sigma_{Q}$ are accounted largely by hyperfine splittings, hence the mass differences scale as $1 / m_{Q}$. Some of recent predictions based on potential quark models can be found in [3]. Few of the theoretical predictions on natural widths have been published [4].

The analysis is based on an integrated luminosity of $6.0 \mathrm{fb}^{-1}$ collected with the CDF II detector between March 2002 and February 2010. The component of the CDF II detector [5] most relevant for this analysis is the tracking system comprising a microvertex silicon detector and a large drift chamber [6]. The trigger [7] on displaced tracks with $p_{T}>2 \mathrm{GeV} / c$ selects a data sample enriched with hadron modes of $b$-quarks produced in the detector.

We study decays in the exclusive fully reconstructed decay channel, $\Sigma_{b}^{(*) \pm} \rightarrow \Lambda_{b}^{0} \pi_{\text {soft }}^{ \pm}, \Lambda_{b}^{0} \rightarrow$ $\Lambda_{c}^{+} \pi_{b}^{-}, \Lambda_{c}^{+} \rightarrow p K^{-} \pi^{+1}$. To reconstruct $\Lambda_{b}^{0}$ signal first the $\Lambda_{c}^{+} \rightarrow p K^{-} \pi^{+}$candidate is built up of three tracks with $p_{T}>0.4 \mathrm{GeV} / c$ and with a fitted common vertex. Then, the $\Lambda_{c}^{+}$candidate

\footnotetext{
${ }^{1}$ Charge-conjugate combinations are always implied unless otherwise stated.
} 
within $\pm 3 \cdot \sigma_{M}$ range around PDG [8] value of $M\left(\Lambda_{c}^{+}\right)$is combined with a pion $\pi_{b}^{-}$- track of $p_{T}>1.5 \mathrm{GeV} / c$ and the $\Lambda_{b}^{0} \rightarrow \Lambda_{c}^{+} \pi_{b}^{-}$candidate is subjected to a single pion $\pi_{b}^{-}$-track vertex fit with the contributing $\Lambda_{c}^{+}$candidate constrained to its PDG [8] mass. Figure 2 with further details provided in its caption shows the reconstructed base $\Lambda_{b}^{0}$ signal. This is currently the largest sample of $\Lambda_{b}^{0}$ decays, with $\sim 16300$ candidates in the signal and with the ratio $S / B \approx 1.8$.

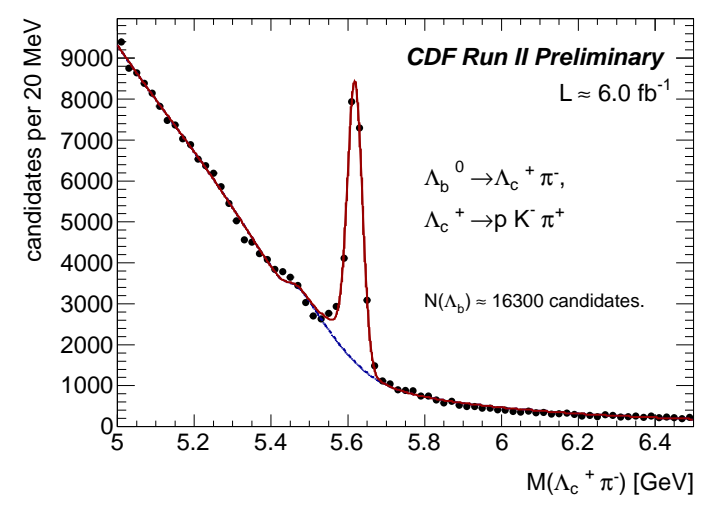

Figure 2: $\Lambda_{b}^{0}$ signal reconstructed using the total statistics of $\int \mathscr{L} d t=6 \mathrm{fb}^{-1}$. In the analysis at least two tracks within $p, K^{-}, \pi^{+}, \pi_{b}^{-}$are demanded to meet the criteria firing the displaced track trigger. The fitted $\Lambda_{b}^{0} \rightarrow \Lambda_{c}^{+} \pi_{\text {soft }}$ - vertex is required to be by at least $12 \cdot \sigma_{c t}$ away from the primary one, where $c t$ is a product of a proper decay time and the speed of light, $\sigma_{c t}$ is its vertex fit uncertainty.

Finally, the $\Lambda_{b}^{0}$ candidates within $\pm 3 \cdot \sigma_{M}$ window around $\Lambda_{b}^{0}$ signal peak are combined with a soft pion track of $p_{T}>0.2 \mathrm{GeV} / c$ and again the $\Sigma_{b}^{(*) \pm} \rightarrow \Lambda_{b}^{0} \pi_{\text {soft }}^{ \pm}$candidates are subjected to a single track vertex fit. The analysis is performed with the $Q$-value distributions, $Q=m\left(\Lambda_{b}^{0} \pi_{\text {soft }}^{ \pm}\right)-$ $m\left(\Lambda_{b}^{0}\right)-m\left(\pi^{ \pm}\right)_{\text {PDG }}$ where the $\Lambda_{b}^{0}$ candidate's resolution and most of the systematic uncertainties are canceled. The signals of $\Sigma_{b}^{(*)-}$ and $\Sigma_{b}^{(*)+}$ are reconstructed as two peaks in the corresponding $Q$ - value spectra and subjected independently to the unbinned likelihood fits. The signal shapes are modeled with a non-relativistic Breit-Wigner function with the width modified by a $P$-wave factor $\left(\frac{p_{\pi}^{*}}{p_{\pi}^{* 0}}\right)^{3}$, where $p_{\pi}^{*}$ is the $\pi_{s o f t}$ momentum in the $\Sigma_{b}^{(*)}$ rest frame, and $p_{\pi}^{* 0}$ is the $\pi_{s o f t}$ momentum in the same rest frame but calculated at the resonance pole mass [9]. The Breit-Wigner function is further convoluted with the detector resolution described by two Gaussians. The background under the signals is described by a formula, specifically $\sqrt{\left(Q+m_{\pi}\right)^{2}-t h r^{2}} \cdot\left(C+b_{1} \cdot Q+b_{2} \cdot\left(2 \cdot Q^{2}-1\right)\right)$, where $t h r$ is fixed to a pion mass $m_{\pi}=0.1396 \mathrm{GeV} / c^{2}$. Figure 3 shows the $\Sigma_{b}^{(*) \pm} Q$-value distributions with the projections of the corresponding unbinned fits superimposed.

The significance of the observed signals is tested against several null hypothesis using the logratio of the minima likelihoods, $-2 \cdot \log \left(\mathscr{L}_{0} / \mathscr{L}_{1}\right)$, reached by the fitter for the base line hypothesis $\mathscr{L}_{1}$ and for a particular null hypothesis $\mathscr{L}_{0}$. Any signal combination is by $\gtrsim 10.0 \sigma$ significant than the sole background model. The most critical null hypothesis is a broad single peak fluctuating to the default two peak model: both $\Sigma_{b}^{(*)-}$ and $\Sigma_{b}^{(*)+}$ observed two-peak signal structures are individually preferred by the base line hypothesis at $\gtrsim 7.0 \sigma$ level relative to such null hypothesis.

The measurement results are listed in a Table 1. The systematic uncertainties come from the residual uncertainty in the mass difference $Q$-value due to a CDF tracking momentum scale, small bias caused by the fitter yielding the natural width results, the uncertainties due to the signal and background models implemented in the fitter. To study the systematic effects related to the detector resolution of our signals we used a large available data sample of $D^{*+} \rightarrow D^{0} \pi^{+}$. The momentum scale dominates the systematics on the fitted pole $Q$-values while the resolution uncertainty determines the systematics of the results on the widths. 

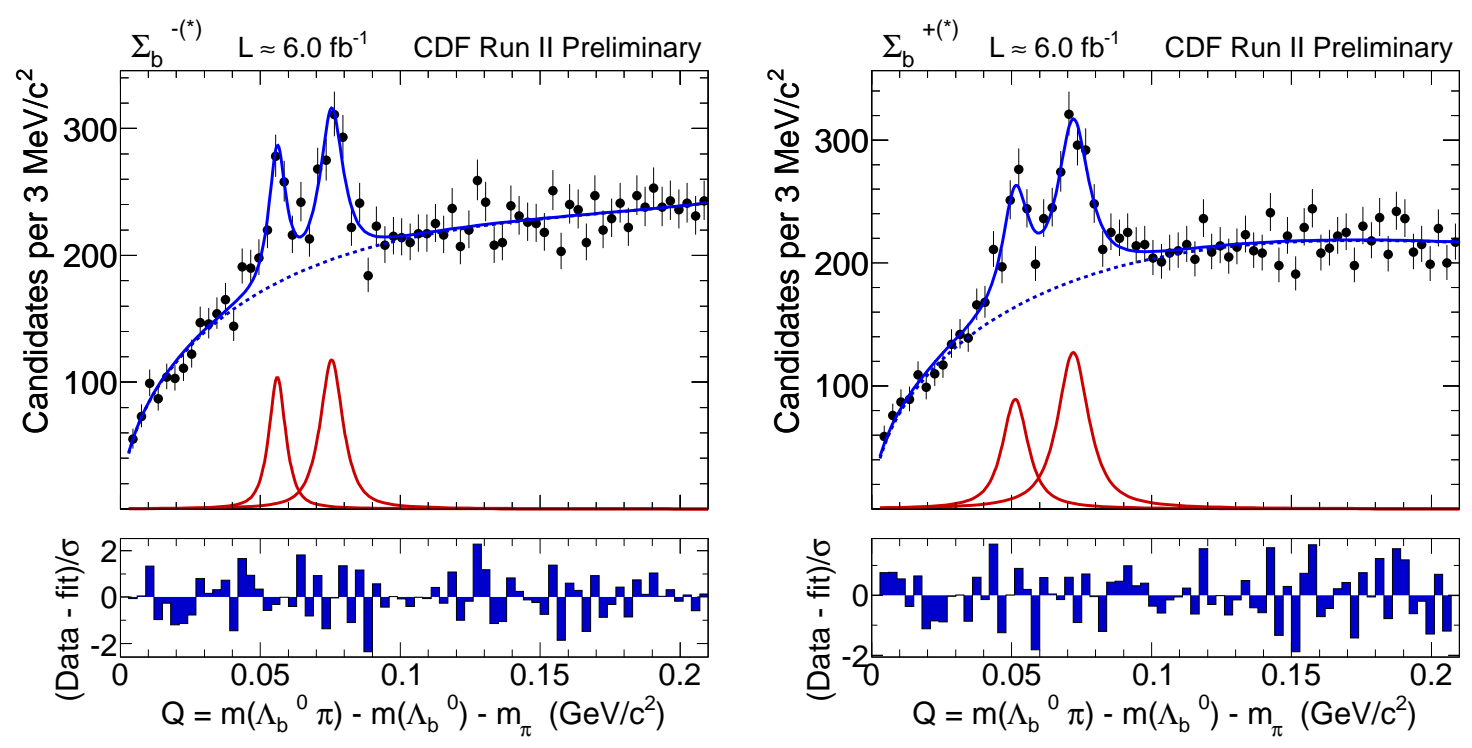

Figure 3: The $Q$-value spectra, where $Q=M\left(\Lambda_{b}^{0} \pi^{ \pm}\right)-M\left(\Lambda_{b}^{0}\right)-m_{\pi^{ \pm}}$, are shown for $\Sigma_{b}^{(*)-}$ (left) and for $\Sigma_{b}^{(*)+}$ (right) candidates with the projection of the corresponding unbinned likelihood fit superimposed.

\begin{tabular}{|c|c|c|c|c|}
\hline State & $\begin{array}{l}Q \text {-value, } \\
\mathrm{MeV} / \mathrm{c}^{2}\end{array}$ & $\begin{array}{c}\text { Absolute Mass, } \\
\mathrm{m}, \mathrm{MeV} / c^{2}\end{array}$ & $\begin{array}{c}\text { Natural Width, } \\
\Gamma, \mathrm{MeV} / c^{2} \\
\end{array}$ & $\begin{array}{c}\text { Yield, } \\
\text { num. of cands. }\end{array}$ \\
\hline$\overline{\Sigma_{b}^{+}}$ & $52.0_{-08-0.4}^{+0.9+0.09}$ & $5811.2_{-0.8}^{+0.9} \pm 1.7$ & $9.2_{-2.9-1.1}^{+3.8+1.0}$ & $468_{-95-15}^{+110+18}$ \\
\hline$\Sigma_{b}^{-}$ & $56.2_{-0.5-0.4}^{+0.8+0.4}$ & $5815.5_{-0.5}^{+0.6} \pm 1.7$ & $4.3_{-2.1-1.1}^{+3.9-1.1}$ & $333_{-73}^{+93} \pm 35$ \\
\hline$\Sigma_{b}^{*+}$ & $72.7 \pm 0.7_{-0.6}^{+0.12}$ & $5832.0 \pm 0.7 \pm 1.8$ & $10.4_{-2.2-1.2}^{+2.7+0.8}$ & $782_{-103-27}^{+114+25}$ \\
\hline$\Sigma_{b}^{*-}$ & $75.7 \pm 0.6_{-0.6}^{+0.08}$ & $5835.0 \pm 0.6 \pm 1.8$ & $6.4_{-1.8-1.1}^{+2.2+0.7}$ & $522_{-76}^{+85} \pm 29$ \\
\hline & \multicolumn{4}{|c|}{ Isospin Mass Splitting, $\mathrm{MeV} / \mathrm{c}^{2}$} \\
\hline $\begin{array}{l}\mathrm{m}\left(\Sigma_{b}^{+}\right)-\mathrm{m}\left(\Sigma_{b}^{-}\right) \\
\mathrm{m}\left(\Sigma_{b}^{*+}\right)-\mathrm{m}\left(\Sigma_{b}^{*-}\right)\end{array}$ & \multicolumn{4}{|c|}{$\begin{array}{c}-4.2_{-0.9-0.09}^{+1.1+0.07} \\
-3.0 \pm 0.9_{-0.13}^{+0.12} \\
\end{array}$} \\
\hline
\end{tabular}

Table 1: Summary of the final results. In all the quoted values the first uncertainty is a statistic one and the second one is a systematic. To extract the absolute masses the best CDF mass measurement for $\Lambda_{b}^{0}[10]$ has been used.

In a conclusion, we have measured the $\Sigma_{b}^{(*) \pm}$ bottom baryons using a sample of $\sim 16300$ $\Lambda_{b}^{0}$ candidates identified in $\Lambda_{b}^{0} \rightarrow \Lambda_{c}^{+} \pi^{-}$mode with the $6 \mathrm{fb}^{-1}$ of data collected with the CDF II detector. More details on the presented analysis can be found in [11]. The first observation of $\Sigma_{b}^{(*) \pm}$ bottom baryons made by CDF Collaboration [1] has been confirmed with the every individual signal reconstructed at a significance of $\gtrsim 7 \sigma$ in Gaussian terms. The direct mass difference measurements have been found with the statistical precision by a factor of $\gtrsim 2.3$ better w.r.t. to the published results [1] and according to the amount of the statistics available. The measurements are in a good agreement with the previously found results [1]. The isospin mass splittings within $I=1$ triplets $\Sigma_{b}$ and $\Sigma_{b}^{*}$ have been extracted for the first time. The precision of the experimental isospin splittings is as good as the ones quoted by PDG [8] for $\Sigma_{c}$ states. The $\Sigma_{b}^{(*)-}$ states have a 
higher mass value than their $\Sigma_{b}^{(*)+}$ partners following a standard pattern for every known isospin multiplet [12] even though excluding their charm partners [8], $\Sigma_{c}$ where the supposedly natural order of masses within isotriplets is still violated [13]. The natural widths of both $\Sigma_{b}^{ \pm}$and $\Sigma_{b}^{* \pm}$ states have been measured for the first time. Given the statistical errors the measurements are in the agreement with the theoretical expectations [ 4 ].

\section{Acknowledgments}

The authors are grateful to their colleagues from the CDF $B$-Physics Working Group for useful suggestions and comments made during preparation of this talk. The authors thank Juan Pablo Fernandez (CIEMAT, Spain) and Sally C. Seidel (Univ. of New Mexico, USA) for a successful cooperation and a support of this work.

\section{References}

[1] T. Aaltonen et al. [CDF Collaboration], Phys. Rev. Lett. 99, 202001 (2007) [arXiv:0706.3868 [hep-ex]].

[2] D. Ebert, R. N. Faustov and V. O. Galkin, Phys. Lett. B 659, 612 (2008) [arXiv:0705.2957 [hep-ph]]; E. E. Jenkins, Phys. Rev. D 54, 4515 (1996) [arXiv:hep-ph/9603449]; E. E. Jenkins, Phys. Rev. D 55, 10 (1997) [arXiv:hep-ph/9609404]; E. E. Jenkins, Phys. Rev. D 77, 034012 (2008) [arXiv:0712.0406 [hep-ph]].

[3] S. Gasiorowicz and J. L. Rosner, Am. J. Phys. 49, 954 (1981); J. L. Rosner, Phys. Rev. D 75, 013009 (2007) [arXiv:hep-ph/0611207]; M. Karliner and H. J. Lipkin, Phys. Lett. B 660, 539 (2008) [arXiv:hep-ph/0611306]; M. Karliner, Nucl. Phys. Proc. Suppl. 187, 21 (2009) [arXiv:0806.4951 [hep-ph]].

[4] J. G. Korner, M. Kramer and D. Pirjol, Prog. Part. Nucl. Phys. 33, 787 (1994) [arXiv:hep-ph/9406359]; X. H. Guo, K. W. Wei and X. H. Wu, Phys. Rev. D 77, 036003 (2008) [arXiv:0710.1474 [hep-ph]].

[5] D. Acosta et al. [CDF Collaboration], Phys. Rev. D 71, 032001 (2005) [arXiv:hep-ex/0412071].

[6] C. S. Hill [On behalf of the CDF Collaboration], Nucl. Instrum. Meth. A 530 (2004) 1; A. A. Affolder et al. [CDF Collaboration], Nucl. Instrum. Meth. A 526, 249 (2004).

[7] E. J. Thomson et al., IEEE Trans. Nucl. Sci. 49, 1063 (2002); J. A. Adelman et al. [CDF Collaboration], Nucl. Instrum. Meth. A 572, 361 (2007).

[8] K. Nakamura et al. [Particle Data Group], J. Phys. G 37 (2010) 075021.

[9] J. D. Jackson, Nuovo Cim. 34, 1644 (1964); J. D. Jackson, private communication, 11.06.07; M. Artuso et al. [CLEO Collaboration], Phys. Rev. D 65, 071101 (2002) [arXiv:hep-ex/0110071]; G. Brandenburg et al. [CLEO Collaboration], Phys. Rev. Lett. 78, 2304 (1997).

[10] D. E. Acosta et al. [CDF Collaboration], Phys. Rev. Lett. 96, 202001 (2006) [arXiv:hep-ex/0508022].

[11] CDF Collaboration, "Measurement of the Bottom Baryon Resonances $\Sigma_{b}$ and $\Sigma_{b}^{*}$, public CDF note 10286, October 14, 2010.

[12] F. K. Guo, C. Hanhart and U. G. Meissner, JHEP 0809, 136 (2008) [arXiv:0809.2359 [hep-ph]].

[13] Felix Wick (for the CDF Collaboration), "Charm baryon spectroscopy at CDF", talk at ICHEP 2010, Paris, 22-28 July 2010, to be published in the Proceedings; see also CDF Collaboration, "Charm Baryon Spectroscopy", public CDF note 10260, August 12, 2010. 